incubation temperature to $37^{\circ} \mathrm{C}$ or up to $50^{\circ} \mathrm{C}$. Under such conditions, the ladders shown here could be obtained within 3 hours rather than within days.

8. C. Gaillard and F. Strauss, data not shown.

9. Cell culture (CV1 line), nuclei purification, protein extraction, gel retardation, as well as phosphocellulose, DNA-sephacryl, and hydroxyapatite chromatography were performed as described by $F$. Strauss and A. Varshavsky [Cell 37, 889 (1984)] Because HMG1 and HMG2 copurified on these columns, a fourth chromatographic step on carboxymethyl-cellulose was performed as described by G. H. Goodwin and E. W. Johns [in Methods in Cell Biology, D. M. Prescott, Ed. (Academic Press, New York, 1977), vol. 16, chap. 15]. In appropriate salt conditions, HMG1 does not bind to this column, whereas HMG2 does.

10. S. Paillard and F. Strauss Nucleic Acids Res. 19, 5619 (1991); T. Mimori, J. A. Hardin, J. A. Steitz, J. Biol. Chem. 261, 2274 (1986)

11. E. W. Johns, Ed., The HMG Chromosomal Proteins (Academic Press, London, 1982); M. Bustin, D. A. Lehn, D. Landsman, Biochim. Biophys. Acta 1049, 231 (1990)

12. J. M. Wright and G. H. Dixon Biochemistry 27, 576 (1988); M. E. Bianchi, M. Beltrame, G. Paonessa
Science 243, 1056 (1989); M. E. Bianchi, L. Falciola S. Ferrari, D. M. J. Lilley, EMBO J. 11, 1055 (1992)

13. D. M. J. Lilley and R. M. Clegg, Annu. Rev. Biophys. Biomol. Struct. 22, 299 (1993).

14. Y. Timsit, E. Westhof, R. P. P. Fuchs, D. Moras, Nature 341, 459 (1989); Y. Timsit and D. Moras, J. Mol. Biol. 221, 919 (1991)

15. D. B. Haniford and D. E. Pulleyblank, J. Biomol. Struct. Dyn. 1, 593 (1983); H. Castleman, L. Specthrie, L. Makowski, B. F. Erlanger, ibid. 2 271 (1984)

16. J. A. Blaho and R. D. Wells, Prog. Nucleic Acid Res. Mol. Biol. 37, 107 (1989)

17. S. McGavin, J. Mol. Biol. 55, 293 (1971); J. H. Wilson, Proc. Natl. Acad. Sci. U.S.A. 76, 3641 (1979).

18. D. M. J. Lilley, Nature 357, 282 (1992); K. Giese, J. Cox, R. Grosschedl, Cell 69, 185 (1992); S. Ferrari et al., EMBO J. 11, 4497 (1992); T. T. Paull, M. J. Haykinson, R. C. Johnson, Genes Dev. 7, 1521 (1993)

19. A 120-bp Cla I-Eco RI restriction fragment that contained a 60 -bp tract of poly(CA).poly(TG) was used. The nucleotide sequence of the poly(CA)containing strand of the fragment was as follows: CGATAAGCT TCTAGAGATCCC-(CA) ${ }_{30}-$ GGG-
ATCCGTCGACTCTAGAGGATCCCCGGGCGAGCTCG.

20. Four-percent polyacrylamide gels were used Two different electrophoresis buffers were used, with identical results [either $40 \mathrm{mM}$ tris-acetate, 20 $\mathrm{mM}$ sodium acetate, $1 \mathrm{mM}$ EDTA ( $\mathrm{pH} 7.8$ ), or 6.7 $\mathrm{mM}$ tris-acetate, $3.3 \mathrm{mM}$ sodium acetate, $1 \mathrm{mM}$ EDTA ( $\mathrm{pH} 7.8$ ) with buffer recirculation]. Identical results were obtained with gels run at $4^{\circ} \mathrm{C}$ or $25^{\circ} \mathrm{C}$.

21. DNA spreading was done with cytochrome $c$ on an aqueous hypophase, followed by rotary shadowing with platinum. A control, in which the same DNA was spread at a 1000-fold higher concentration without preincubation at high ionic strength to form complexes, failed to show $X$-shaped structures, confirming that these are not due to random crossing of DNA molecules on the electron microscope grid.

22. We thank $L$. Jonk, who participated in an early stage of this work, C. Antony for help with the electron microscopy facility, and S. Elsevier for critical reading of the manuscript. Supported by grants from the Association Française des Myopathes, the Association pour la Recherche contre le Cancer, and the Ligue Nationale Française Contre le Cancer.

14 December 1993; accepted 7 March 1994

\section{A Cell Cycle Regulator Potentially Involved in Genesis of Many Tumor Types}

\author{
Alexander Kamb, ${ }^{*}$ Nelleke A. Gruis, Jane Weaver-Feldhaus, \\ Qingyun Liu, Keith Harshman, Sean V. Tavtigian, \\ Elisabeth Stockert, Rufus S. Day III, Bruce E. Johnson, \\ Mark H. Skolnick
}

A putative tumor suppressor locus on the short arm of human chromosome 9 has been localized to a region of less than $\mathbf{4 0}$ kilobases by means of homozygous deletions in melanoma cell lines. This region contained a gene, Multiple Tumor Suppressor 1 (MTS1), that encodes a previously identified inhibitor (p16) of cyclin-dependent kinase 4. MTS1 was homozygously deleted at high frequency in cell lines derived from tumors of lung, breast, brain, bone, skin, bladder, kidney, ovary, and lymphocyte. Melanoma cell lines that carried at least one copy of MTS1 frequently carried nonsense, missense, or frameshift mutations in the gene. These findings suggest that MTS1 mutations are involved in tumor formation in a wide range of tissues.
The genetics of cancer involves positive regulators of the transformed state (oncogenes) as well as negative regulators (tumor suppressor genes). More than 100 oncogenes have been characterized, and although less than a dozen tumor suppressor genes have been identified to date, the number is expected to increase beyond 50 (1). The involvement of so many genes Harshman, S. V. Tavtigian, Myriad Genetics, Inc., Salt Lake City, UT 84108, USA.

E. Stockert, Ludwig Institute for Cancer Research Memorial Sloan-Kettering Cancer Center, New York NY 10021, USA.

R. S. Day III, Department of Medicine, Cross Cancer Institute, Edmonton, Alberta, Canada.

B. E. Johnson, Lung Cancer Biology Section, National Cancer Institute, Bethesda, MD 20889, USA

M. H. Skolnick, Myriad Genetics, Inc., Salt Lake City, UT 84108, and Department of Medical Informatics, University of Utah Medical Center, Salt Lake City, UT 84108, USA.

*To whom correspondence should be addressed.
A. Kamb, N. A. Gruis, J. Weaver-Feldhaus, Q. Liu, K underscores the complexity of the growth control mechanisms that maintain the integrity of normal tissue. This complexity is manifested in another way. So far no single gene has been shown to participate in the development of all or even the majority $\Phi^{\circ}$ human cancers. The most common oncot genic mutations are in HRAS, found in 10 t $15 \%$ of solid tumors (2). The most frequento ly mutated tumor suppressor gene is in the p53 gene, mutated in roughly $50 \%$ of a tumors (3). Without a target that is commo to all transformed cells, the dream of "magic bullet" that can destroy or revere. cancer cells while leaving normal tissue un harmed is improbable. The hope for a new generation of specifically targeted antitumou drugs may rest on the ability to identifop tumor suppressor genes or oncogenes thac play general roles in control of cell division

One of the mechanisms for controllin tumor growth might involve direct regulae tion of the cell cycle. Genes that controw the decision to initiate DNA replication at attractive candidates for oncogenes or tug mor suppressor genes depending on whethed they have a stimulatory or inhibitory role it the process. Indeed, several oncogenes an tumor suppressor genes have been found 10 participate directly in the cell cycle. For instance, one of the cyclins, a class of proteins that promotes DNA replication
Table 1. Deletions in tumor cells and primary tumors.

\begin{tabular}{lccc}
\hline Tumor type & Lines $(n)$ & Deletions $(n)$ & Deletions (\%) \\
\hline Astrocytoma & 17 & 14 & 82 \\
Bladder & 15 & 5 & 33 \\
Breast & 10 & 6 & 60 \\
Colon & 20 & 0 & 0 \\
Glioma & 35 & 25 & 71 \\
Leukemia & 4 & 1 & 25 \\
Lung & 59 & 15 & 25 \\
Melanoma & 99 & 57 & 58 \\
Neuroblastoma & 10 & 0 & 0 \\
Osteosarcoma & 5 & 3 & 60 \\
Ovary & 7 & 2 & 29 \\
Renal & 9 & 5 & 56 \\
Total & 290 & 133 & 46 \\
\hline
\end{tabular}


and mitosis, has been implicated as an oncogene (4), and the retinoblastoma tumor suppressor is a substrate of the cyclindependent protein kinases (cdks) (5).

A hallmark of tumor suppressor genes is that they are deleted at high frequency in certain tumor types. The deletions often involve loss of a single allele, so-called loss of heterozygosity (LOH), but may also involve homozygous deletion of both alleles. For $\mathrm{LOH}$, the remaining allele is presumed to be nonfunctional, either because of a preexisting inherited mutation or because of a secondary somatic mutation.

The human 9p21 region contains chromosomal inversions, translocations, heterozygous deletions, and homozygous deletions in glioma cell lines, non-small cell lung cancer lines, leukemia lines, and melanoma lines $(6-8)$. Certain 9p21 markers are deleted in more than half of all melanoma lines (9). These findings suggest that 9p21 contains a tumor suppressor locus that may be involved in genesis of several tumor types. In a previous study, we reported the results of a YAC and P1 chromosomal walk in a region of $9 \mathrm{p} 21$ (9). This work produced a physical map and a set of sequence tagged sites (STSs) that were used to analyze nearly 100 melanoma cell lines for homozygous deletions. More than half of these cell lines contained homozygous deletions that clustered around a single cosmid, c5 (Fig. 1A). Fine structure mapping experiments with STSs derived from c5 revealed the presence of small, nonoverlapping, homozygous deletions of $\mathrm{c} 5$ sequences in six melanoma cell lines. On the basis of this result, it was probable that a tumor suppressor gene lay at least partly within cosmid c5.

To search for candidate tumor suppressor genes, the DNA sequence of parts of cosmid $\mathrm{c} 5$ was determined (10). When this

Fig. 1. Maps of cosmid c5 region. These maps are part of a larger physical map derived from a chromosomal walk in the region (1). (A) Relevant STSs used for the deletion analysis are shown, as are cosmids and P1s mentioned in the text. The c1.b marker lies proximal to P1 1062 and is not shown. The transcriptional orientations of MTS1 and MTS2 are shown by arrows. (B) Restriction map and STS map of cosmid c5. Positions of coding exons for MTS1 and MTS2 are shown as thick bars. E1 and E2, coding exon 1 and coding exon 2, respectively; B, Bam HI; S, Sal I; R1, Eco Rl; and R5, Eco RV. (C) Deletions in tumor cell lines of STSs. Positive controls and negative controls were included in every polymerase chain reaction (PCR) experiment and cell lines in which only one or two of the STSs were deleted (such as class 20) were retested at least twice. The cell lines used for this study and their class designations are available from the authors upon request. + , presence of DNA; -, absence (homozygous deletion). sequence was compared with sequences in GenBank, two distinct regions of c5 were identified that were similar to a region of a previously defined gene encoding human cdk4 inhibitor, or p16 (11). These two sequences were named Multiple Tumor Suppressor 1 (MTS1) and MTS2 (Fig. 1B).

Detailed comparison of genomic sequence from c5 with the p16 mRNA sequence revealed that MTS1 contained a stretch of $307 \mathrm{bp}$ that was identical to a portion of the p16 coding sequence. This stretch of nucleotides in MTS1 was flanked by recognizable splice junction sequences. Further characterization of MTS1 showed that it included the entire coding sequence of p16 plus two introns. The two introns divided the coding sequence of p16 into three regions: a 5 ' region of $126 \mathrm{bp}$ (coding exon 1), a middle region of $307 \mathrm{bp}$ (coding exon 2), and a $3^{\prime}$ region of $11 \mathrm{bp}$ (coding exon 3).

MTS2 contained a region of DNA sequence $93 \%$ identical to p16 sequence

A

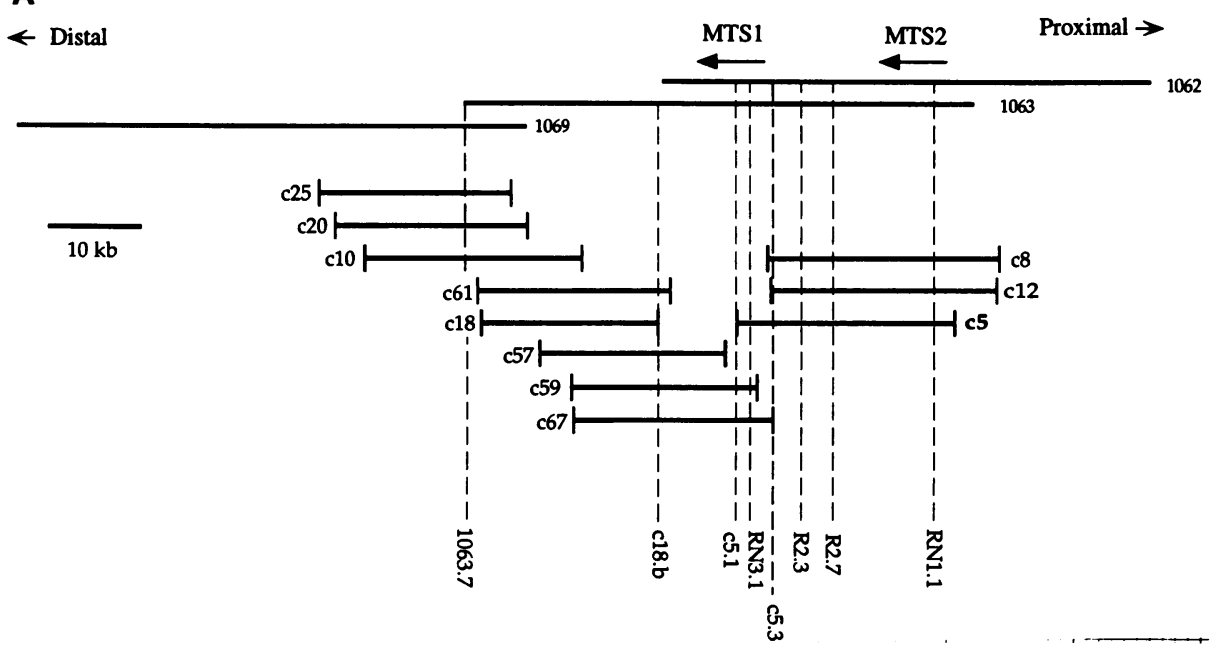

B

$\leftarrow$ Distal $\quad$ Proximal $\rightarrow$

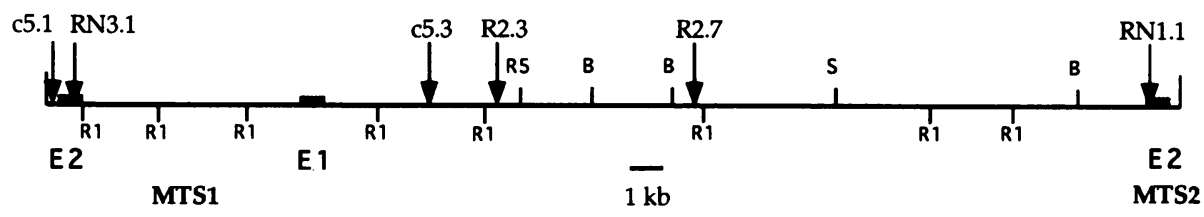

C

\begin{tabular}{|c|c|c|c|c|c|c|c|c|c|c|}
\hline Class & \# lines & 1063.7 & c18.B & c5.1 & RN3.1 & c5.3 & R2.3 & R2.7 & RN1.1 & c1.B \\
\hline 1 & 57 & - & - & - & - & - & - & - & - & - \\
\hline 2 & 5 & + & - & - & - & - & - & - & - & - \\
\hline 3 & 1 & + & + & - & - & - & - & - & - & - \\
\hline 4 & 3 & + & + & + & + & - & - & - & - & - \\
\hline 5 & 23 & - & - & - & - & - & - & - & - & + \\
\hline 6 & 8 & + & - & - & - & - & - & - & - & + \\
\hline 7 & 4 & + & + & - & - & - & - & - & - & + \\
\hline 8 & 2 & + & + & + & + & - & - & - & - & + \\
\hline 9 & 7 & - & - & - & - & - & - & - & + & + \\
\hline 10 & 1 & + & + & - & - & - & - & - & + & + \\
\hline 11 & 1 & + & + & + & - & - & - & - & + & + \\
\hline 12 & 1 & + & + & + & + & - & - & - & + & + \\
\hline 13 & 2 & - & - & - & - & - & - & + & + & + \\
\hline 14 & 4 & - & - & - & - & - & + & + & + & + \\
\hline 15 & 6 & + & - & - & - & - & + & + & + & + \\
\hline 16 & 1 & + & + & - & - & - & + & + & + & + \\
\hline 17 & 1 & - & - & - & - & + & + & + & + & + \\
\hline 18 & 4 & + & + & - & - & + & + & + & + & $t^{\circ}$ \\
\hline 19 & 1 & + & 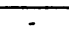 & - & + & + & + & + & + & + \\
\hline 20 & 1 & + & + & - & + & + & + & + & + & + \\
\hline 21 & 157 & + & + & + & + & + & + & + & + & + \\
\hline
\end{tabular}

Deletions

Total

94

125

126

115

113

103

66 
which extended from the $5^{\prime}$ end of coding exon 2 roughly $255 \mathrm{bp}$ toward intron 2 . By analogy with MTS1, we refer to this region in MTS2 as coding exon 2. The coding exon 2 sequences of MTS1 and MTS2 diverged abruptly at a point $50 \mathrm{bp}$ upstream of intron 2 in MTS1 (Fig. 2). No obvious 5' splice sequence was found at the position equivalent to the $5^{\prime}$ splice junction of intron 2 in the MTS1 gene. A stop codon occurred in the open reading frame of coding exon 2 at the codon immediately following the divergence point. Thus, if the proteins encoded by MTS1 and MTS2 were identical in size upstream of the divergence point, and if an alternative splice site upstream of the divergence point were not used, the MTS2 product would be 20 residues shorter than p16. The sequence similarity between MTS1 and MTS2 also extended nearly 40 nucleotides upstream from the $3^{\prime}$ splice junction of intron 1 . Thus, portions of presumptive noncoding DNA were as conserved as some areas of presumptive coding DNA (12).

The occurrence of two closely related genes on cosmid c5 suggested that other related genes might exist in the region. To test this possibility, Southern blots were prepared from restriction enzyme digests of cosmids c5, c12, c59, P1s 1063 and 1062, and human genomic DNA (Fig. 1A). These blots were probed with a fragment containing most of exon 2 from MTS1, including the region shared with MTS2. Two Eco RI frag- ments and two Hind III fragments were detected with the probe in both cloned DNA and genomic DNA (Fig. 3). Because coding exon 2 sequences from MTS1 and MTS2 do not contain Eco RI or Hind III sites, this result was consistent with the presence of only two p16-like genes in the genome, MTS1 and MTS2. A second Southern blot identical to the first was probed with coding exon 1 from MTS1. Only a single hybridizing fragment was seen in genomic DNA and in cloned DNA (13). This suggested that MTS2 does not contain an exon that closely resembles coding exon 1 of MTS1.

Because of the high frequency of deletions at $9 \mathrm{p} 21$ in multiple tumor types, we analyzed cell lines derived from 12 different types of tumor for deletions of MTS1, MTS2, or both. A set of STSs located in or around MTS1 and MTS2 was used to test genomic DNA from tumor cell lines for the presence or absence of the expected fragment (Fig. 1) (14). Lack of amplification of the predicted STS fragment from cell line genomic DNA was interpreted as indicative of homozygous deletion of the specific STS in that cell line. Homozygous deletions of at least one marker were detected in all tumor types tested other than neuroblastoma lines and colon tumor lines (Table 1). Excluding these types, the percentage of deletions varied from $25 \%$ in lung cancer and leukemia lines to $82 \%$ in astrocytomas. Collectively, 133 of 290 tumor lines contained deletions of at least one marker in the region tested.
This number represented a minimum estimate of the percentage of tumor lines that harbor homozygous deletions, because the STSs used for the analysis did not completely cover the two genes. Thus, certain small deletions could escape detection. In addition, lesions such as insertions or deletions of a few nucleotides, and nucleotide substitutions, would be missed by this approach.

To improve the estimate of the total number of cell lines containing MTS1 or MTS2 mutations, a set of melanoma cell lines that did not contain obvious homozygous deletions of MTS1 or MTS2 sequences were examined more closely for genetic lesions. Genomic DNA sequences from coding exons 1 and 2 of MTS1, comprising $97 \%$ of the coding sequence, were amplified and screened for polymorphisms (15). Eighteen mutations, distributed in 14 of 34 melanoma lines, were observed (Table 2). Three of these mutations were frameshifts, seven were nonsense mutations, four were missense mutations, and four were silent. Three of the four lines that contained silent mutations also contained additional mutations and 16 of 18 mutations were located in coding exon 2. All but one line contained exclusively hemi- or homozygous polymorphisms, suggesting that the other homologous chromosomes had incurred de-

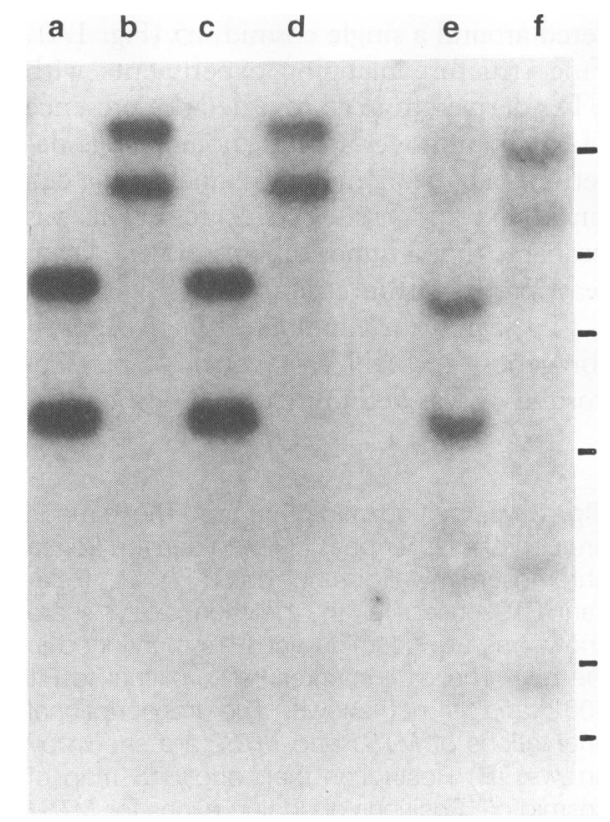

Fig. 3. Southern blot of $P 11062$ (a and b), $P 1$ 1063 (c and d), and genomic DNA (e and f) digested with Eco RI (a, c, and e) and Hind III (b, d, and f). P1 DNA (500 pg) and $5 \mu \mathrm{g}$ of human genomic DNA were blotted from a $0.7 \%$ agarose gel. Size markers in kilobases from top to bottom of the autoradiogram are: $23.1,9.4$, $6.6,4.4,2.3$, and 2.0. The probe was a 142-bp PCR product that contained nucleotides 98 to 240 of MTS1 coding exon 2 (Fig. 2). Hybridization was performed with standard procedures (19). The final wash was in $0.1 \times$ SSPE, $0.1 \%$ SDS at $50^{\circ} \mathrm{C}$ for $15 \mathrm{~min}$.
Fig. 2. DNA sequence alignment of MTS1 and MTS2. Regions of sequence identity between MTS1 and MTS2 are shown in boldface. The positions of the $3^{\prime}$ splice junction of intron 1 and $5^{\prime}$ splice junction of intron 2 for MTS 1 are shown by triangles. The divergence point near the $3^{\prime}$ end of coding exon 2 is indicated by an arrow. 
letions. The single line that was heterozygous contained two nonsilent mutations, a finding consistent with the view that each homolog had undergone independent mutational events. Based on this DNA sequence and deletion analysis of MTS1, a minimum of $75 \%$ melanoma lines contained mutant MTS1 or had lost the gene from both homologs.

A similar analysis of coding exon 2 from MTS2 did not reveal any polymorphisms. In addition, no deletions unequivocally involved MTS2 alone (Fig. 1C). When $\mathrm{RN} 1.1$, the marker within MTS2, was deleted, markers within MTS1 were also lost with five exceptions (classes 4, 8, and 12 in Fig. 1C). However, two of these exceptional lines were shown to have MTS1 mutations or deletions: melanoma line SK-MEL-26 (class 8) was missing coding exon 1 from MTS1 (13) and melanoma line SK-MEL-12 (class 8) contained a hemizygous missense mutation in coding exon 2 of MTS1 (Table 2) (13). The remaining three lines apparently did contain coding exon 1 of MTS1; however, it is possible that the regulatory region of MTS1 was affected by the deletion breakpoints located upstream. Thus, the function of MTS2 has not been resolved. The striking sequence similarity between MTS1 and MTS2 implies that MTS2 may also inhibit one or more cdks, perhaps including cdk4. The majority of homozygous deletions removed both MTS1 and MTS2. Thus, MTS2 may have some role in tumorigenesis. Alternatively, MTS2 may be a nonfunctional gene or may have a role entirely different from MTS1.

It is possible that mutation or loss of MTS1 is a product of cell growth in culture. However, a high percentage of primary leukemia cells also contain homozygous de- letions of the $\alpha$-interferon gene cluster, a gene family located less than $500 \mathrm{~kb}$ from MTS1 (8). Studies of melanoma cell lines suggest that deletions of $\boldsymbol{\alpha}$-interferon genes invariably involve markers that extend beyond MTS1 toward the centromere (13). In addition, LOH studies of human primary, non-small cell lung cancer and primary head and neck carcinoma have demonstrated that the minimal area of heterozygous deletion in these tumors encompasses $9 \mathrm{p} 21$ 22 (8). Finally, nonsense and splice-junction mutations in MTS1 have been observed in primary melanomas and bladder tumors (16). Because deletions of the 9p21 region and nonsense mutations of MTS1 occur in primary tumor cells as well as cultured cell lines, the deletions observed in tumor cell lines are unlikely to be purely a result of cell growth in culture.

Dividing eukaryotic cells must pass through two critical decision points: the $\mathrm{G}_{1}-\mathrm{S}$ transition where DNA synthesis commences and the $G_{2}-M$ transition where mitosis begins. The machinery that controls cell division has multiple components, many of which are related (16). The cdks may be at the heart of the control apparatus in that they regulate by phosphorylation a number of key substrates that in turn trigger the transition from $G_{1}$ to $S$ and from $G_{2}$ to $M$. So far, four types of cdk have been defined (cdk2-5) that may participate in $\mathrm{G}_{1}-\mathrm{S}$ control as well as a set of positive regulators of these cdks (for example, cyclins C, D1-3, E). Recently several negative regulators have also been identified, including p21 (p20 in mouse), and the MTS1 gene product p16 $(11,17,18)$. On the basis of in vitro studies and on its interaction with p53, p21 has been proposed as a general inhibitor of all cdks (18). Thus, in vitro, p16 appears

Table 2. Mutations in melanoma lines determined by DNA sequence analysis.

\begin{tabular}{|c|c|c|c|}
\hline Cell line & Mutation & Coding effect & Location $\dagger$ \\
\hline SK-MEL-61 & $\begin{array}{l}\mathrm{G} \rightarrow \mathrm{A} \\
\mathrm{G} \rightarrow \mathrm{A}\end{array}$ & $\begin{array}{l}\text { None } \\
\text { Gly } \rightarrow \text { Ser }\end{array}$ & $\begin{array}{l}258 \\
259\end{array}$ \\
\hline SK-MEL-21 & $\mathrm{C} \rightarrow \mathrm{T}$ & Arg $\rightarrow$ stop & 166 \\
\hline SK-MEL-17 & $\mathrm{G} \rightarrow \mathrm{A}$ & Ala $\rightarrow$ Thr & 436 \\
\hline SK-MEL-112 & 5-base deletion & Frameshift & $284-288$ \\
\hline SK-MEL-178 & $\mathrm{C} \rightarrow \mathrm{T}$ & Arg $\rightarrow$ stop & 232 \\
\hline SK-MEL-124 & $\mathrm{G} \rightarrow \mathrm{A}$ & Trp $\rightarrow$ stop & 324 \\
\hline SK-MEL-86 & $\mathrm{C} \rightarrow \mathrm{T}$ & Arg $\rightarrow$ stop & 232 \\
\hline SK-MEL-131 & $\begin{array}{l}\text { 8-base deletion } \\
\mathrm{C} \rightarrow \mathrm{A}\end{array}$ & $\begin{array}{l}\text { Frameshift } \\
\text { None }\end{array}$ & $\begin{array}{c}166-173 \\
165\end{array}$ \\
\hline SK-MEL-150(het)* & $\begin{array}{l}\mathrm{C} \rightarrow \mathrm{T} \\
\mathrm{C} \rightarrow \mathrm{T} \\
\mathrm{C} \rightarrow \mathrm{T}\end{array}$ & $\begin{array}{l}\text { Pro } \rightarrow \text { Leu } \\
\text { None } \\
\text { Arg } \rightarrow \text { stop }\end{array}$ & $\begin{array}{l}335 \\
231 \\
232\end{array}$ \\
\hline SK-MEL-12 & $C \rightarrow T$ & Pro $\rightarrow$ Leu & 335 \\
\hline SK-MEL-156 & $\mathrm{C} \rightarrow \mathrm{T}$ & None & 372 \\
\hline SK-MEL-101 & $\mathrm{G} \rightarrow \mathrm{A}$ & Trp $\rightarrow$ stop & 323 \\
\hline SK-MEL-158 & $\mathrm{C} \rightarrow \mathrm{T}$ & Gin $\rightarrow$ stop & 142 \\
\hline SK-MEL-145 & 2-base deletion & Frameshift & $122-123$ \\
\hline
\end{tabular}

*Het stands for heterozygote and refers to the presence in the sample of both the wild-type and mutant sequence. TThe nucleotide positions of the base changes (location) derive from the numbering scheme used in Serrano et al. (11).

more specific than $\mathrm{p} 21$. Both of these inhibitors are expected to antagonize entry into $S$ phase.

If p16 inhibits cdk4 in vivo, cdk4 and its cyclin partners are strong candidates for oncogenes that could be major factors in tumorigenesis. The prevalence of mutations in the p16 gene suggests the possibility that cdk4 may serve as a general activator of cell division in most, if not all, cells. Further biochemical studies of the effects of p16 on different cdks may help clarify the hierarchy of cdk activity in both normal cells and transformed cells. Proof of our model awaits direct demonstration of the tumor suppressor function of $\mathrm{p} 16$ and of the interaction between p16 and cdk4 in vivo.

\section{REFERENCES AND NOTES}

1. A. G. Knudson, Nature Genet. 5, 103 (1993).

2. J. A. Anderson, J. C. Irish, B. Y. Ngan, J. Otolaryngol. 21, 321 (1992).

3. C. C. Harris, Science 262, 1980 (1993)

4. G. A. Lammie et al., Oncogene 6, 439 (1991); T. Motokura et al., Nature 350, 512 (1991); C. L. Rosenberg et al., Proc. Natl. Acad. Sci. U.S.A. 88 9638 (1991); D. A. Withers et al., Mol. Cell. Biol. 11, 4846 (1991).

5. M. E. Ewen et al., Cell 73, 487 (1993)

6. O. I. Olopade et al., Cancer Res. 52, 2523 (1992); R. Lukeis, L. Irving, M. Gason, S. Hasthorpe, Genes Chromosomes Cancer 2, 116 (1990); M. O. Diaz et al., Proc. Natl. Acad. Sci. U.S.A. 85, 5259 (1988); P. G. Middleton et al., Leukemia 5, 680 (1991); J. W. Fountain et al., Proc. Natl. Acad. Sci. U.S.A. 89 , 10557 (1992); O. I. Olopade et al., Cancer Res. 53, 2410 (1993); J. Q. Cheng, S. C. Jhanwar, Y. Y. Lu, J. R. Testa, ibid., p. 4761; C. D. James, J. He, V. P. Collins, M. J. Allalunis-Turner, R. S. Day, ibid., p. 3674.

7. M. O. Diaz et al., N. Engl. J. Med. 322, 77 (1990).

8. A. Merlo, E. Gabrielson, F. Askin, D. Sidransky, Cancer Res. 54, 640 (1994); P. van der Riet et al., ibid., p. 1156.

9. J. Weaver-Feldhaus et al., Proc. Natl. Acad. Sci. U.S.A., in press.

10. DNA sequence determination of $c 5$ was carried out by subcloning the internal Hind III fragments and Eco RI fragments and determining the DNA sequences of these subclones independently. Primer sequence walking steps were carried out sequentially on each subcloned template. DNA sequencing reactions were performed with the PRISM Ready Reaction DyeDeoxy Terminator Cycle Sequencing kit (ABI), and the products were analyzed on an $A B I 373$ sequencer.

11. M. Serrano, G. J. Hannon, D. Beach, Nature 366, 704 (1993).

12. To exclude the possibility that the sequence divergence in coding DNA might be a cloning artifact, we designed PCR primers to amplify specifically across the sequence divergence point of MTS2. These primers amplified a fragment of the predicted size from cosmid, P1, and genomic DNA (15). Therefore the divergent sequence located near the $3^{\prime}$ end of exon 2 in MTS2 is bona fide genomic sequence.

13. A. Kamb et al., unpublished data

14. These STSs included two markers on the distal side of MTS1 (1063.7 and c18.b), two markers within MTS1 (c5.1, located between coding exons 2 and 3 , and RN3.1, located within coding exon 2), three markers between MTS1 and MTS2 (c5.3, R2.3, and R2.7), one marker within MTS2 (RN1.1, located at the $3^{\prime}$ end of coding exon 2), and one marker on the proximal side of MTS2 (c1.b). The method for preparation of cell line DNA has been described (9). The PCR conditions used for STS amplification were one cycle at $95^{\circ} \mathrm{C}(5 \mathrm{~min})$; four cycles at $95^{\circ} \mathrm{C}(10 \mathrm{~s})$ with 
the annealing temperature $\left(T_{\text {ann }}\right)=68^{\circ} \mathrm{C}(10 \mathrm{~s})$ and $72^{\circ} \mathrm{C}(10 \mathrm{~s})$; four cycles with $T_{\text {ann }}=66^{\circ} \mathrm{C}$; four cycles with $T_{\text {ann }}=64^{\circ} \mathrm{C}$; four cycles with $T_{\text {ann }}=62^{\circ} \mathrm{C}$; and 30 cycles with $T_{1 n}=60^{\circ} \mathrm{C}$. The DNA sequences of primers used for the STS analysis were 1063.7F, CCGTTTCAGCTTCTCATCAC; 1063.7R, CCGACTGTCCCATTGTGATT; c18.bF, CAAAGACTTTATGGATGGGG; c18.bR, TCCATTTCTCTGCTTGCTC; c5.1F, GAAGTCTTGGTCCTGATGTC; c5.1R, CTCTTCTGCACAACTCAACT; RN3.1F, GGATAGAGAACTCAAGAAGG; RN3.1R, TCTGAGCTTTGGAAGCTCT; c5.3F, GTGGTAGAACTAGGACAGGG c5.3R, CTGTGTTAAGCCTTCATAGA; R2.3F, GAAAATGAAACTGTACCCATTG; R2.3R, GGGACACACATTAAATACACT; R2 7F GAGAACAGGTTTTGGGCAG; R2.7R, AACTAGACCTAGGGATAAGG; c1.bF, AAGCTTTCCCACAAACTGGC; c1.bR, AATGCCTTGGCATAAGGGAC

15. Fragments for DNA sequence determination were amplified as described (14), except that $5 \%$ dimethyl sulfoxide was added to the reaction. The products were purified from a $1.2 \%$ agarose gel with Qiaex beads (QIAGEN). Genomic fragments corresponding to coding exons 1 or 2 from MTS 1 or coding exon 2 from MTS2 were amplified with specific primers and analyzed by cycle sequencing with $\left[\alpha-P^{32}\right]$ deoxyadenosine triphosphate (19). Products were run on $6 \%$ polyacrylamide gels. All $A$ reactions were loaded side by side followed by the $C$ reactions, and so on. Detection of polymorphisms was by eye with confirmation on the other strand. The DNA sequences of the primers used for amplification and sequence determination were

Coding exon 1, MTS1. Amplification: 2F, GAAGAAAGAGGAGGGGCTG; 1108R, GCGCTACCTGATTCCAATTC. Sequencing: 1108R.

Coding exon 2, MTS1. Amplification: 42F, GGAAATTGGAAACTGGAAGC; 551R, TCTGAGCTTTGGAAGCTCT. Sequencing: $42 \mathrm{~F}$ and 551P. Coding exon 2, MTS2. Amplification: 89F, TGA
GTTTAACCTGAAGGTGG: 50R, GGGTGGGAAATTGGGTAAG. Sequencing: 89F and 50R.

16. N. A. Gruis et al., in preparation.

17. J. W. Harper, G. R. Adami, N. Wei, K. Keyomarsi, S. J. Elledge, Cell 75, 805 (1993); Y. Gu, C. W. Turck, D. O. Morgan, Nature 366, 707 (1993); W. S. el-Deiry, T. Tokino, V. E. Velculescu, Cell 75, 817 (1993)

18. Y. Xiong et al., Nature 366, 701 (1993).

19. J. Sambrook, E. F. Fritsch, T. Maniatis, Molecular Cloning: A Laboratory Manual (Cold Spring Harbor Laboratory Press, Cold Spring Harbor, NY, 1989)

20. We thank D. Ballinger, $S$. Bayer, J. Collet, $P$. Dayananth, W. Ding, C. Frye, C. Hussey, M McClure, D. Shattuck-Eidens, S. Stone, and T. Tran for suggestions and assistance. Supported in part by $\mathrm{NIH}$ grant CA-48711.

17 March 1994; accepted 1 April 1994

\section{AAAS-Newcomb Cleveland Prize To Be Awarded for a Report, Research Article, or an Article Published in Science}

The AAAS-Newcomb Cleveland Prize is awarded to the author of an outstanding paper published in Science. The value of the prize is $\$ 5000$; the winner also receives a bronze medal. The current competition period began with the 4 June 1993 issue and ends with the issue of 27 May 1994.

Reports, Research Articles, and Articles that include original research data, theories, or syntheses and are fundamental contributions to basic knowledge or technical achievements of far-reaching consequence are eligible for consideration for the prize. The paper must be a first-time publication of the author's own work. Reference to pertinent earlier work by the author may be included to give perspective.

Throughout the competition period, readers are invited to nominate papers appearing in the $\mathrm{Re}$ ports, Research Articles, or Articles sections. Nominations must be typed, and the following information provided: the title of the paper, issue in which it was published, author's name, and a brief statement of justification for nomination. Nominations should be submitted to the AAASNewcomb Cleveland Prize, AAAS, Room 924, 1333 H Street, NW, Washington, DC 20005, and must be received on or before 30 June 1994. Final selection will rest with a panel of distinguished scientists appointed by the editor of Science.

The award will be presented at the 1995 AAAS annual meeting. In cases of multiple authorship, the prize will be divided equally between or among the authors. 